\title{
Hybrid Next-Fit Algorithm for the Two-Dimensional Rectangle Bin-Packing Problem
}

\author{
J. B. G. Frenk, Rotterdam, and G. Galambos, Szeged
}

Received August 4, 1986

\begin{abstract}
Zusammenfassung
Hybrid Next-Fit Algorithm for the Two-Dimensional Rectangle Bin-Packing Problem. We present a new approximation algorithm for the two-dimensional bin-packing problem. The algorithm is based on two one-dimensional bin-packing algorithms. Since the algorithm is of next-fit type it can also be used for those cases where the output is required to be on-line (e. g. if we open an new bin we have no possibility to pack elements into the earlier opened bins). We give a tight bound for its worst-case and show that this bound is a parameter of the maximal sizes of the items to be packed. Moreover, we also present a probabilistic analysis of this algorithm.
\end{abstract}

Key words: Two-dimensional packing, bin-packing, heuristic algorithm, worst-case analysis, probabilistic analysis, on-line algorithm.

Hybrid Next-Fit Algorithmus für das zweidimensionale Rechteck-Packungsproblem. Wir geben einen neuen Näherungs-Algorithmus für das zweidimensionale Packungsproblem an. Er beruht auf zwei eindimensionalen Packungsalgorithmen. Da der Algorithmus von next-fit Typ ist, kann er auch in solchen Fällen benutzt werden, wo die Ausgabe on-line sein muß (d. h. sobald wir einen neuen Behälter eröffnen, haben wir keine Möglichkeit, Elemente in früher geöffnete Behälter zu packen). Wir geben eine gute Schranke im schlechtesten Fall an und zeigen, daß diese Schranke von der Maximalgröße der gepackten Rechtecke abhängt. Schließlich untersuchen wir noch das mittlere Verhalten des Algorithmus.

\section{Introduction}

During the last decade a wide variety of fast heuristics have been developed for the one-dimensional bin-packing problem. This problem can be stated as follows: We are given a list $L=\left\{p_{1}, p_{2}, \ldots, p_{n}\right\}$ of $n$ objects (or items) with sizes $s\left(p_{i}\right), i=1, \ldots, n$, and bins, each with a positive integer capacity of $C,\left(0<s\left(p_{i}\right) \leq C, i=1, \ldots, n\right)$. What is the smallest integer $m$ such that there is a partition $L=B_{1} \cup B_{2} \cup \ldots \cup B_{m}$ satisfying $\sum_{p_{i} \in B_{j}} s\left(p_{i}\right) \leq C$ ? We usually think of each list of $B_{j}$ as being the contents of a bin of capacity $C$, and attempt to minimize the number of bins needed for a packing of $L$.

It is known that the bin-packing problem belongs to the class of $N P$-hard problems (see Garey and Johnson [5]). So there is no efficient algorithm to solve it, unless $P=N P$. 
Therefore there were numerous heuristics developed to solve this problem. To decide on an algorithm whether it is better than another one there are different methods.

A possibility to analyze an algorithm is to examine its worst-case behavior. Since we use this method, we define the so-called asymptotic performance ratio which characterizes the worst-case behavior of an algorithm. For any bin packing algorithm $A$, let $A(L)$ denote the number of bins needs to pack $L$ by the algorithm $A$, and OPT $(L)$ denotes the number of bins used by an optimal packing. Let

$$
R_{A}(k)=\sup \left\{\frac{A(L)}{k} \mid \operatorname{OPT}(L)=k\right\},
$$

and let us define the asymptotic performance ratio $R_{A}$ as the largest limit of a convergent subsequence of $R_{A}(k)$, i.e.

$$
R_{A}=\limsup _{k \rightarrow \infty} R_{A}(k) .
$$

In applications we often have a bound $0<r \leq C$ for the size of the items of the list $L$. This means that for all $p_{i} \in L$ the size $s\left(p_{i}\right) \leq r$. In this case we denote the asymptotic performance ratio by $R_{A}\langle r\rangle$.

We will now present four types of algorithms to which we will refer later. The interested readers find details in Baker and Coffman [1], Johnson [6] and Johnson et al. [7].

The Next-Fit (NF) algorithm first places the elements into the bin $B_{1}$. Suppose that $p_{i}$ is now to be packed, and let $B_{j}$ be the highest inexed non-empty bin. The algorithm places $p_{i}$ into $B_{j}$ if it will fit (e.g. it is not allowed to pack the element into the bins $\left.B_{j}, j<i\right)$, otherwise open a new bin $\left(B_{j+1}\right)$ placing $p_{i}$ into it $\left(R_{\mathrm{NF}}=2\right)$.

The First-Fit (FF) algorithm places each successive piece into the lowest indexed bin of the sequence $B_{1}, B_{2}, \ldots$ into which it will fit $\left(R_{\mathrm{FF}}=17 / 10\right)$.

We note that the main difference between the above two algorithms is that according to FF it is generally possible for a piece to be packed to the left of the rightmost occupied bin, but the NF fills the bins in sequence e.g. $B_{1}, B_{2}, \ldots, B_{i-1}$ receive no further pieces after the first piece is packed in $B_{i}$.

These algorithms do not know the items in advance. If we have the possibility to order the elements before using the algorithm we would get better results. The FirstFit Decreasing (FFD) and the Next-Fit Decreasing (NFD) differ from the above ones only in the preordering the items $\left(R_{\mathrm{FFD}}=11 / 9, R_{\mathrm{NFD}}=1.691 \ldots\right)$.

The one-dimensional bin-packing problem is well-studied. Relatively few results have been published on the two-dimensional rectangle bin-packing. The problem is the following: We are given a list $L$ of rectangles. The size of a rectangle $p \in L$ is given by an ordered pair of width and height $(w(p), h(p))$, and we are given rectangular bins with sizes $W$ and $H$. We have to pack the rectangles into a minimal number of bins so that 
a) the sides of the rectangles are parallel the corresponding sides of the bins (no rotation allowed);

b) no two rectangles in a bin overlap.

Chung, Garey and Johnson [2] developed an algorithm to give an approximative solution of this problem. They called it Hybrid-First Fit (HFF) because the algorithm mixes the FFD and FF rules. They proved that

$$
\frac{182}{90} \leq R_{\mathrm{HFF}} \leq \frac{17}{8} .
$$

A tight bound for $R_{\mathrm{HFF}}$ is not known. Actually, as far as we know there is no heuristic algorithm with acceptable tight bound for the two-dimensional bin packing problem. The other feature of HFF is that it has an off-line output in the sense that it supposes that whenever an element is to be placed all open bins can be used to pack it. But there are numerous applications where we do not have this possibility, i.e. if we pack an element in a new bin, we are lost the old ones for further packing (on-line output). Such problems can arise in computer science in time-dependent sequential storage allocations, in some computer network problems, packing shelves systems, filling of a cold-storage plant and so on. So in this case one can not use the above mentioned algorithm to get a fast approximative solution.

In this paper we give an algorithm with time complexity $O(n \log n)$ for the twodimensional rectangle bin-packing problem. Since this algorithm uses the results concerning the one-dimensional algorithms, see Baker and Coffman [1] and Johnson [6], it has an on-line output. We prove a tight asymptotic bound for it in Section 2. Moreover, we also present in Section 3 a probabilistic analysis of this algorithm, which we call Hybrid-Next Fit (HNF).

\section{The HNF Bound}

First of all we present the HNF algorithm.

Step 1: Order the rectangles $p$ of the list in nonincreasing direction according to their heights $h(p)$.

Step 2: Take out the first item, say $p$, from the list and place it in the first bin into the lower left hand corner. Let us call the rectangular area of height $h(p)$ of the bin whose left most part of width $w(p)$ is covered by $p$ the block opened by $p$.

Step 3: Take the next rectangle of $L$ and try to place it into the last opened block. If this is impossible then open a new block (as defined in 2.) within the current bin if this is possible. If there is no space for the new block in the current bin open a new bin with a new first block.

Step 4: If we have items unplaced then goto Step 3, else stop.

Note that without loss of generality we can assume in the sequel that the bin heights $H$ and widths $W$ are equal and $H=W=1$.

Since we will examine the worst case behavior of our algorithm we have to define the asymptotic performance ratio for the two-dimensional case as well. Let $r, s$ be 
integers such that

If

$$
\begin{aligned}
& \text { I. } \frac{1}{r+1}<\max _{p \in L} w(p) \leq \frac{1}{r} \text {. } \\
& \text { II. } \frac{1}{s+1}<\max _{p \in L} h(p) \leq \frac{1}{s} \text {. }
\end{aligned}
$$

$$
R_{A}\langle k, r, s\rangle=\sup \left\{\frac{A(L)}{k} \mid \operatorname{OPT}(L)=k\right\},
$$

then the asymptotic performance ratio is

$$
R_{A}\langle r, s\rangle=\limsup _{k \rightarrow \infty} R_{A}\langle k, r, s\rangle .
$$

During the proofs of our claims - see below - we shall use sequences which came up first in number theory, but they have also been used frequently to solve different one-dimensional bin-packing problems (see Baker and Coffman [1] and Liang [9]). For an integer $s \geq 1$ let

$$
t_{1}(s)=s+1, \quad t_{i+1}(s)=\prod_{j=1}^{i} t_{j}+1 \quad i \geq 1 .
$$

We shall use two simple results concerning these sequences (see Baker and Coffman [1])

$$
\begin{gathered}
\sum_{i=1}^{\infty} \frac{1}{t_{i}(s)}=\frac{2}{s+1}, \\
\frac{2}{s+1}-\sum_{i=1}^{k-1} \frac{1}{t_{i}(s)}=\frac{1}{t_{k}(s)-1} .
\end{gathered}
$$

Our main result is a theorem concerning the asymptotic performance ratio of the HNF algorithm.

Theorem 2.1: Let $L$ be a set (or list) of rectangles, which satisfies conditions $I$ and II. Let

Then

$$
\begin{gathered}
\gamma_{s}=\sum_{i=1}^{\infty} \frac{1}{t_{i}(s)-1}, \quad \gamma_{s}^{*}=\frac{s-1}{s}+\gamma_{s}, \\
\alpha= \begin{cases}2, & \text { if } r=1 ; \\
r, & \text { otherwise. }\end{cases}
\end{gathered}
$$

$$
R_{\mathrm{HNF}}\langle r, s\rangle=\frac{\alpha}{\alpha-1} \gamma_{s}^{*} .
$$

Proof: The proof relies on the proof which has been given for the one-dimensional case by Baker and Coffman [1], but now we use our two-dimensional weight function.

This statement of the theorem immediately follows from the following two lemmas. 
Lemma 2.2: For any list $L$ which satisfies the conditions $I$ and II the following inequality is true

$$
\operatorname{HNF}(L) \leq \frac{\alpha}{\alpha-1} \gamma_{s}^{*} \mathrm{OPT}(L)+9 .
$$

Lemma 2.3: There is a sequence of lists $L_{\tau}(\tau=1,2 \ldots)$ for which each list $L$ satisfies the conditions $I$ and $I I$, and

$$
\lim _{\tau \rightarrow \infty} \frac{\operatorname{HNF}\left(L_{\tau}\right)}{\operatorname{OPT}\left(L_{\tau}\right)}=\frac{\alpha}{\alpha-1} \gamma_{s}^{*} .
$$

Proof: We first prove Lemma 2.2. Let us call an interval $\left(\frac{1}{k+1}, \frac{1}{k}\right]$ a $\gamma_{s}$-interval if $k=t_{i}(s)-1$ for some $i$. Rectangles whose heights are in a $\gamma_{s}$-interval will be called $\gamma_{s}$-pieces. Define a weight function $W_{s}(p)$ as follows: for any rectangle $p \in L$, $h(p) \in\left(\frac{1}{k+1}, \frac{1}{k}\right], k \geq s$,

$$
W_{s}(p)= \begin{cases}\frac{\alpha}{\alpha-1} w(p) \frac{1}{k}, & \text { if } p \text { is a } \gamma_{s} \text {-piece; } \\ \frac{\alpha}{\alpha-1} w(p) h(p) \frac{k+1}{k}, & \text { otherwise. }\end{cases}
$$

During the proof we shall use the following, easily provable, statements concerning the weight function $W_{s}(p)$.

Corollary 2.4: The weight function $W_{s}(p)$ is a nondecreasing function of height for the items with equal width of $p$. Furthermore, it is strictly increasing, except the $\gamma_{s}$-intervals, where it is constant.

Corollary 2.5: The function $\frac{W_{s}(p)}{h(p) w(p)}$ decreases monotonically in $\gamma_{s}$-intervals, but it is constant in any other interval for the items with equal width.

Corollary 2.6: The weight function $W_{s}(p)$ is additive in vertical direction. That means, if we have a piece with sizes $h(p)$ and $w(p)$, and we divide it with a "vertical" line (i.e. parallel to its height) into two pieces $p_{1}, p_{2}$ of sizes $h(p), w\left(p_{1}\right)$ and $h(p), w\left(p_{2}\right)$ then

$$
W_{s}(p)=W_{s}\left(p_{1}\right)+W_{s}\left(p_{2}\right) .
$$

For a set of $Q, Q \subseteq L$, let $W_{s}(Q)=\sum_{p \in Q} W_{s}(p)$. We shall prove that the above defined weighting function has two properties.

(1) $W_{s}(L) \geq \operatorname{HNF}(L)-9$.

(2) $W_{s}(L) \leq \frac{\alpha}{\alpha-1} \gamma_{s}^{*} \operatorname{OPT}(L)$.

The desired result follows immediately from these two properties. 


\section{Claim 2.7:}

$$
W_{s}(L) \geq \operatorname{HNF}(L)-9 .
$$

Proof: Our proof consists of two steps. First we derive a list $\bar{L}$ from the original list $L$ in such a way that we disregard the fact that pieces of smaller height than the first item may occur within a block (see Fig. 2.1).
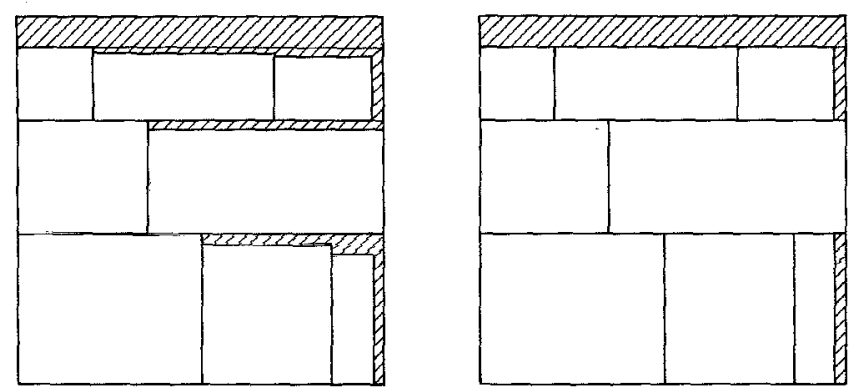

Fig. 2.1

Let $\bar{L}^{\prime}$ denote the list derived from the list $\bar{L}$ by removing the items which have been packed into the last bin of the HNF packing of $L$. In the first step we prove that

$$
W_{s}(\bar{L})>\operatorname{HNF}(\bar{L})-\frac{13}{3} \text {. }
$$

Let us suppose that the bin $B_{i}$ belongs to the HNF packing of $\bar{L}^{\prime}$.

Case A: Let us suppose that $B_{i}$ contains exactly $k_{i}$ blocks all of whose heights are in the interval $\left(\frac{1}{k_{i}+1}, \frac{1}{k_{i}}\right]$. If we denote the $j$-th block in the $i$-th bin by $C_{i, j}$, then

$$
\begin{aligned}
W_{s}\left(B_{i}\right) & \geq \frac{1}{k_{i}} \sum_{j=1}^{k_{i}} \frac{\alpha}{\alpha-1} \sum_{p \in C_{i, j}} w(p) \\
& =\frac{1}{k_{i}} \sum_{j=1}^{k_{i}}\left[\frac{\alpha}{2(\alpha-1)} \sum_{p \in C_{i, j}} w(p)+\frac{\alpha}{2(\alpha-1)} \sum_{p \in C_{i, j}} w(p)\right] \\
& >\frac{k_{i}-1}{k_{i}}+\frac{1}{k_{i}} \frac{\alpha}{2(\alpha-1)} \sum_{p \in C_{i, 1}} w(p)+\frac{1}{k_{i}} \frac{\alpha}{2(\alpha-1)} \sum_{p \in C_{i, k_{i}}} w(p) .
\end{aligned}
$$

This expression is valid whether or not $\left(\frac{1}{k_{i}+1}, \frac{1}{k_{i}}\right]$ is a $\gamma_{s}$-interval. We will refer to
this type of bins as A-type bins.

Case B: Let us suppose that $B_{i}$ is not an A-type bin. In this case the bin contains at least two blocks with heights in different intervals. These bins are called transition bins (see Baker and Coffman [1]).

Case B.1: Let us suppose that $B_{i}$ is a transition bin and contains at least one block whose height belongs to a $\gamma_{s}$-interval $\left(\frac{1}{k_{i}+1}, \frac{1}{k_{i}}\right]\left(k_{i} \geq s+2\right)$. By definition of the 
HNF rule the cumulative height of the blocks in $B_{i}$ is at least $\frac{k_{i}-1}{k_{i}}$. Let us denote the height of the block $C_{i, j}$ by $h\left(C_{i, j}\right)\left(j=1, \ldots, n_{i}\right)$ and use the inequality

Then we get

$$
W_{s}(p) \geq \frac{\alpha}{\alpha-1} h(p) w(p) \text {. }
$$

$$
\begin{aligned}
W_{s}\left(B_{i}\right) & \geq \sum_{j=1}^{n_{i}}\left[\frac{\alpha}{\alpha-1} h\left(C_{i, j}\right) \sum_{p \in C_{i, j}} w(p)\right] \\
& \geq \sum_{j=2}^{n_{i}} h\left(C_{i, j}\right)+\frac{\alpha}{2(\alpha-1)} h\left(C_{i, 1}\right) \sum_{p \in C_{i, 1}} w(p)+\frac{\alpha}{2(\alpha-1)} h\left(C_{i, n_{i}}\right) \sum_{p \in C_{i, n_{i}}} w(p) .
\end{aligned}
$$

We will refer to this type of bins as B1-type bins.

Case B.2: Let us suppose that $B_{i}$ is transition bin containing no block whose height is in a $\gamma_{s}$-interval. We suppose again that the smallest block-height is in the interval $\left(\frac{1}{k_{i}+1}, \frac{1}{k_{i}}\right]$. Since the cumulative height of the blocks in $B_{i}$ is at least $\frac{k_{i}-1}{k_{i}}$ and

we get

$$
W_{s}(p) \geq \frac{k_{i}+1}{k_{i}} \frac{\alpha}{\alpha-1} h(p) w(p),
$$

$$
\begin{aligned}
& W_{s}\left(B_{i}\right) \geq \sum_{j=1}^{n_{i}}\left[\frac{k_{i}+1}{k_{i}} \frac{\alpha}{\alpha-1} h\left(C_{i, j}\right) \sum_{p \in C_{i, j}} w(p)\right] \\
&>\frac{k_{i}+1}{k_{i}} \sum_{j=2}^{n_{i}} h\left(C_{i, j}\right)+\frac{k_{i}+1}{k_{i}} \frac{\alpha}{2(\alpha-1)} h\left(C_{i, 1}\right) \sum_{p \in C_{i, 1}} w(p)+ \\
&+\frac{k_{i}+1}{k_{i}} \frac{\alpha}{2(\alpha-1)} h\left(C_{i, n_{i}}\right) \sum_{p \in C_{i, n_{i}}} w(p) .
\end{aligned}
$$

We will refer to this type of bins as B2-type bins.

Let $i_{0}$ be the smallest index for which $B_{i_{0}}$ contains at least two blocks. Let us divide the list $\bar{L}^{\prime}$ into two parts: $\bar{L}_{1}^{\prime}$ contains those elements from $\bar{L}^{\prime}$ which have been packed in bins with one block, $\bar{L}_{2}^{\prime}$ contains the rest of the list.

Consider a bin $B_{i}$ for which $i<i_{0}$. It has to be an A-type bin; moreover in that special case $s=1$ and $k_{i}=1$. So if $i<i_{0}$ then $W_{s}\left(B_{i}\right)=2 \sum_{p \in B_{i}} w(p)$. Therefore if we get two successive bins of this type then $W_{s}\left(B_{i}\right)+W_{s}\left(B_{i+1}\right)>2$. If we combine these bins for all $i<i_{0}$ and $i_{0}$ is even at most one bin remains, so

$$
W\left(\bar{L}_{1}^{\prime}\right)>\operatorname{HNF}\left(\bar{L}_{1}^{\prime}\right)-1 .
$$

Now consider the case $i>i_{0}$. Because of the HNF algorithm the following inequality is true:

$$
\frac{\alpha}{2(\alpha-1)}\left[\sum_{p \in C_{i+1,1}} w(p)+\sum_{p \in C_{i, n_{i}}} w(p)\right]>1 .
$$


Introduce the following notations with the help of (2.2)-(2.4)

$$
\begin{aligned}
& F_{i}= \begin{cases}\frac{k_{i}-1}{k_{i}}, & \text { if } B_{i} \text { is an A-type bin; } \\
\sum_{j=2}^{n_{i}} h\left(C_{i, j}\right), & \text { if } B_{i} \text { is a B1-type bin; } \\
\frac{k_{i}+1}{k_{i}} \sum_{j=2}^{n_{i}} h\left(C_{i, j}\right), & \text { if } B_{i} \text { is a B2-type bin. }\end{cases} \\
& G_{i}= \begin{cases}\frac{1}{k_{i}} \frac{\alpha}{2(\alpha-1)} \sum_{p \in C_{i, 1}} w(p), & \text { if } B_{i} \text { is an A-type bin; } \\
\frac{\alpha}{2(\alpha-1)} h\left(C_{i, 1}\right) \sum_{p \in C_{i, 1}} w(p), & \text { if } B_{i} \text { is a B1-type bin; } \\
\frac{k_{i}+1}{k_{i}} \frac{\alpha}{2(\alpha-1)} h\left(C_{i, 1}\right) \sum_{p \in C_{i, 1}} w(p), & \text { if } B_{i} \text { is a B2-type bin. }\end{cases} \\
& H_{i}= \begin{cases}\frac{1}{k_{i}} \frac{\alpha}{2(\alpha-1)} \sum_{p \in C_{i, k_{i}}} w(p), & \text { if } B_{i} \text { is an A-type bin; } \\
\frac{\alpha}{2(\alpha-1)} h\left(C_{i, n_{i}}\right) \sum_{p \in C_{i, n_{i}}} w(p), & \text { if } B_{i} \text { is a B 1-type bin; } \\
\frac{k_{i}+1}{k_{i}} \frac{\alpha}{2(\alpha-1)} h\left(C_{i, n_{i}}\right) \sum_{p \in C_{i, n_{i}}} w(p), & \text { if } B_{i} \text { is a B2-type bin. }\end{cases}
\end{aligned}
$$

Then the following inequality holds

$$
W\left(\bar{L}_{2}^{\prime}\right) \geq \sum_{i=i_{0}}^{\operatorname{HNF}\left(\bar{L}^{\prime}\right)}\left(F_{i}+G_{i}+H_{i}\right)>\sum_{i=i_{0}+1}^{\text {HNF }\left(\bar{L}^{\prime}\right)}\left(F_{i}+G_{i}+H_{i-1}\right) .
$$

We consider three cases for $B_{i}$.

Case 1: $B_{i}$ is an A-type bin. Then we have to consider three different subcases.

Subcase 1.1: $B_{i-1}$ is an A-type bin. Then

$$
\begin{aligned}
F_{i}+G_{i}+H_{i-1}= & \frac{k_{i}-1}{k_{i}}+\frac{1}{k_{i}} \frac{\alpha}{2(\alpha-1)} \sum_{p \in C_{i, 1}} w(p) \\
& +\frac{1}{k_{i-1}} \frac{\alpha}{2(\alpha-1)} \sum_{p \in C_{i-1, n_{i}-1}} w(p) \\
> & 1 .
\end{aligned}
$$


Subcase 1.2: $B_{i-1}$ is a B2-type bin. Then

$$
\begin{aligned}
F_{i}+G_{i}+H_{i-1}= & \frac{k_{i}-1}{k_{i}}+\frac{1}{k_{i}} \frac{\alpha}{2(\alpha-1)} \sum_{p \in C_{i, 1}} w(p) \\
& +\frac{k_{i-1}+1}{k_{i-1}} \frac{\alpha}{2(\alpha-1)} h\left(C_{i-1, n_{i}-1}\right) \sum_{p \in C_{i-1, n_{i}-1}} w(p) \\
> & 1 .
\end{aligned}
$$

Subcase 1.3: $B_{i-1}$ is a B1-type bin. Then

$$
\begin{aligned}
F_{i}+G_{i}+H_{i-1}= & \frac{k_{i}-1}{k_{i}}+\frac{1}{k_{i}} \frac{\alpha}{2(\alpha-1)} \sum_{p \in C_{i, 1}} w(p) \\
& +\frac{\alpha}{2(\alpha-1)} h\left(C_{i-1, n_{i}-1}\right) \sum_{p \in C_{i-1, n_{i}-1}} w(p) \\
> & \frac{k_{i}-1}{k_{i}}+\frac{1}{k_{i}+1}>1-\frac{1}{k_{i}^{2}} \geq 1-\frac{1}{k_{i-1}^{2}} .
\end{aligned}
$$

Since a B1-type bin is a transition bin, at most two such B1-type bins may occur with blocks of heights in the same $\gamma_{s}$-interval. So the total weight-shortfall is not greater than $2 \sum \frac{1}{k^{2}}$, where we summarize over all $k$ for which the interval $\left(\frac{1}{k+1}, \frac{1}{k}\right]$ is a $\gamma_{s}$-interval. So the cumulative shortfall for these bins is at most

$$
2 \sum_{i=2}^{\infty} \frac{1}{\left(t_{i}(s)-1\right)^{2}}<\frac{5}{6}
$$

Case 2: $B_{i}$ is a B1-type bin. Then for any type of $B_{i-1}$ bin

$$
\begin{aligned}
F_{i}+G_{i}+H_{i-1} \geq & \sum_{j=2}^{n_{i}} h\left(C_{i, j}\right)+\frac{\alpha}{2(\alpha-1)} h\left(C_{i, 1}\right) \sum_{p \in C_{i, 1}} w(p)+ \\
& +\frac{\alpha}{2(\alpha-1)} h\left(C_{i-1} n_{i-1}\right) \sum_{p \in C_{i-1, n_{i-1}}} w(p) \geq \\
> & 1-\frac{1}{k_{i}} .
\end{aligned}
$$

So the cumulative weight of the items in a B 1-type bin is at least $1-\frac{1}{k_{i}}$, where $k_{i}$ is that integer for which the smallest block-height of $B_{i}$ is the interval $\left(\frac{1}{k_{i}+1}, \frac{1}{k_{i}}\right]$. Since a B1-type is a transition bin so at most two B1-type bins may occur with blocks of heights in the same $\gamma_{s}$-interval. Therefore the total weight-shortfall for the B1-type bins is not greater than $2 \sum \frac{1}{k}$, where the sum over all $k$ for which the 
interval $\left(\frac{1}{k+1}, \frac{1}{k}\right]$ is a $\gamma_{s}$-interval. Since the function $\gamma_{s}^{*}$ is a monotone decreasing function of $s$ we get that the cumulative weight-shortfall for these bins is

$$
2 \sum_{i=2}^{\infty} \frac{1}{t_{i}(s)-1}=2\left(\gamma_{s}-\frac{1}{s}\right) \leq 2\left(\gamma_{s}^{*}-1\right)<\frac{3}{2} \text {. }
$$

The last inequality we get from $\gamma_{s}^{*} \leq \gamma_{i}^{*}=1.691 \ldots<\frac{7}{4}$.

Case 3: $B_{i}$ is a B2-type bin. Then

$$
\begin{aligned}
F_{i}+G_{i}+H_{i-1} \geq & \frac{k_{i}+1}{k_{i}} \sum_{j=2}^{n_{i}} h\left(C_{i, j}\right)+\frac{\alpha}{2(\alpha-1)} \frac{k_{i}+1}{k_{i}} h\left(C_{i, 1}\right) \sum_{p \in C_{i, 1}} w(p)+ \\
& +h\left(C_{i-1} n_{i-1}\right) \frac{\alpha}{2(\alpha-1)} \sum_{p \in C_{i-1, n_{i-1}}} w(p) \\
> & \frac{k_{i}^{2}-1}{k_{i}^{2}}-\frac{k_{i}+1}{k_{i}}\left(1-\frac{\alpha}{2(\alpha-1)}\right) h\left(C_{i, 1}\right) \sum_{p \in C_{i, 1}} w(p)+ \\
& +h\left(C_{i-1, n_{i-1}}\right) \sum_{p \in C_{i-1, n_{i-1}}} w(p) \\
> & \frac{k_{i}^{2}-1}{k_{i}^{2}}+\frac{1}{k_{i}}-\frac{k_{i}+1}{k_{i}} \frac{1}{k_{i-1}} \\
\geq & 1-\frac{2}{k_{i}^{2}} .
\end{aligned}
$$

This means that the cumulative weight of the items in $B_{i}$ is at least $1-\frac{2}{k_{i}^{2}}$, where $k_{i}$ is that integer to which the smallest block-height in $B_{i}$ is in the interval $\left(\frac{1}{k_{i}+1}, \frac{1}{k_{i}}\right)$. Since the smallest block-height in $B_{i}$ is not greater than $\frac{1}{s+3}$, we get for the cumulative shortfall of the weights of the B2-type bins

$$
\sum_{k \geq s+3} \frac{2}{k^{2}} \leq 2\left(\frac{\pi^{2}}{6}-\frac{49}{36}\right) \leq \frac{2}{3} .
$$

Summing over all bins in $\bar{L}_{2}^{\prime}$ we get

$$
W_{s}\left(\bar{L}_{2}^{\prime}\right)>\sum_{i=i_{0}+1}^{H N F\left(\bar{L}^{\prime}\right)}\left(F_{i}+G_{i}+H_{i-1}\right)>\operatorname{HNF}\left(\bar{L}_{2}^{\prime}\right)-3 .
$$

From (2.5) and (2.12)

$$
W_{s}\left(\bar{L}^{\prime}\right)=W_{s}\left(\bar{L}_{1}^{\prime}\right)+W_{s}\left(\bar{L}_{2}^{\prime}\right)>\mathrm{HNF}\left(\bar{L}^{\prime}\right)-4 .
$$

Since $W_{s}(\bar{L}) \geq W_{s}\left(\bar{L}^{\prime}\right)$ and $\operatorname{HNF}(\bar{L})=\operatorname{HNF}\left(\bar{L}^{\prime}\right)+1$, therefore

$$
W_{s}(\bar{L})>\operatorname{HNF}(\bar{L})-5 \text {. }
$$


Up to this point we ignored the fact that within a block there may occur pieces with smaller heights than the height of the block. Since the rectangles are ordered according to their heights it is easy to prove that the total sum over all bins of the areas above the rectangles within a block can be bounded by $h\left(C_{1,1}\right)$. Since $h\left(C_{1,1}\right) \leq \frac{1}{S} \leq 1$ we get that the value of the weight-shortfall - returning to the original list - can be bounded by the cummulative weight of pieces in one bin. Using the result of the Claim 2.8 we obtain

and so

$$
W_{s}(\bar{L})<W_{s}(L)+4
$$

$$
W_{s}(L)>\operatorname{HNF}(L)-9 \text {. }
$$

This completes the proof of Claim 2.7.

Claim 2.8: In any packing of $L$ the cumulative weight of the items in any of the bins is at most $\frac{\alpha}{\alpha-1} \gamma_{s}^{*}$. Hence

$$
W_{s}(L) \leq \frac{\alpha}{\alpha-1} \gamma_{s}^{*} \operatorname{OPT}(L) .
$$

Proof: Consider a bin $B$ in an arbitrary packing of $L$. Divide the bin $B$ into bands by vertical lines along the left- and the right-hand sides of all items in it. Denote the $i$-th band by $D_{i}$, and its width by $w\left(D_{i}\right)$ (see Fig. 2.2).

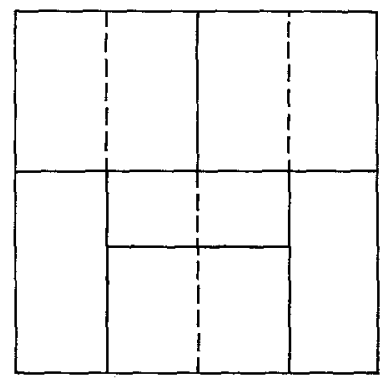

Fig. 2.2

We prove that the cumulative weight of the items (or their segments) within a band is not greater than $\frac{\alpha}{\alpha-1} \gamma_{s}^{*} w\left(D_{i}\right)$. Using Corollary 2.6

$$
W_{s}(B) \leq \frac{\alpha}{\alpha-1} \gamma_{s}^{*} \sum_{D_{i} \in B} w\left(D_{i}\right) \leq \frac{\alpha}{\alpha-1} \gamma_{s}^{*} .
$$

Let us consider the band $D_{i}$. First suppose that there are $s-1$ pieces with heights in the largest $\gamma_{s}$-interval. Their cumulative weight is $\frac{\alpha}{\alpha-1} w\left(D_{i}\right) \frac{s-1}{s}$, and the height occupied by these pieces is at least $\frac{s-1}{s+1}$. So the sum of the heights of the re- 
maining pieces is at most $1-\frac{s-1}{s+1}=\frac{2}{s+1}$. Let $q_{1}, \ldots, q_{m}$ these pieces and $h\left(q_{1}\right) \geq h\left(q_{2}\right) \geq \ldots \geq h\left(q_{m}\right)$. If $h\left(q_{j}\right)$ is in the $j$-th interval $1 \leq j \leq m$ then

$$
W_{s}\left(D_{i}\right)=\frac{\alpha}{\alpha-1} w\left(D_{i}\right)\left[\frac{s-1}{s}+\sum_{j=1}^{m} \frac{1}{t_{j}(s)-1}\right] \leq \frac{\alpha}{\alpha-1} \gamma_{s}^{*} w\left(D_{i}\right) .
$$

Thus assume that there is at least one $q_{j}$, whose height is not in the $j$-th $\gamma_{s}$-interval. Let $k$ be the smallest index of the items of this type. The total weight of the largest $k-1$ pieces is

$$
\frac{\alpha}{\alpha-1} w\left(D_{i}\right) \sum_{j=1}^{k-1} \frac{1}{t_{j}(s)-1} .
$$

The remaining height is not greater than

Since $h\left(q_{k}\right) \geq \frac{1}{t_{k}(s)}$ we get

$$
\frac{2}{s+1}-\sum_{j=1}^{k-1} \frac{1}{t_{j}(s)}=\frac{1}{t_{k}(s)-1} .
$$

$$
\frac{W_{s}\left(q_{l}\right)}{h\left(q_{l}\right)} \leq \frac{\alpha}{\alpha-1} w\left(D_{i}\right) \frac{t_{k}(s)+1}{t_{k}(s)}
$$

for all $l \geq k$. So the cumulative weight of the pieces in the remaining part of the bin is

$$
\begin{aligned}
W_{s}^{\prime}\left(D_{i}\right) & \leq \frac{\alpha}{\alpha-1} w\left(D_{i}\right) \frac{t_{k}(s)+1}{t_{k}(s)} \frac{1}{t_{k}(s)-1} \\
& \leq \frac{\alpha}{\alpha-1} w\left(D_{i}\right)\left(\frac{1}{t_{k}(s)-1}+\frac{1}{t_{k+1}(s)-1}\right) .
\end{aligned}
$$

Therefore

$$
W_{s}\left(D_{i}\right) \leq \frac{\alpha}{\alpha-1} w\left(D_{i}\right)\left(\frac{s-1}{s}+\sum_{j=1}^{k+1} \frac{1}{t_{j}(s)-1}\right)<\frac{\alpha}{\alpha-1} \gamma_{s}^{*} w\left(D_{i}\right) .
$$

Finally let us consider the case that there are only $u \leq s-2$ pieces with heights in the first $\gamma_{s}$-interval. These occupy $\frac{u}{s+1}$ height in the band $D_{i}$, and their total weight is $\frac{\alpha}{\alpha-1} w\left(D_{i}\right) \frac{u}{s}$. So the remaining height in the band is at most $1-\frac{u}{s+1}$. It is clear that the height of the highest item in this part of the band is not greater than $\frac{1}{s+1}$.
Thus

$$
\begin{aligned}
W_{s}\left(q_{j}\right) & \leq \max _{1 \leq j \leq m} \frac{W_{s}\left(q_{j}\right)}{h\left(q_{j}\right)} \sum_{j=1}^{m} h\left(q_{j}\right) \\
& \leq \frac{\alpha}{\alpha-1} w\left(D_{i}\right) \frac{s+2}{s+1}\left(1-\frac{u}{s+1}\right),
\end{aligned}
$$


and so

$$
W_{s}\left(D_{i}\right) \leq \frac{\alpha}{\alpha-1} w\left(D_{i}\right)\left[\frac{s-2}{s}+\frac{3(s+2)}{(s+1)^{2}}\right]<\frac{\alpha}{\alpha-1} \gamma_{s}^{*}
$$

We now prove the Lemma 2.3. It is sufficient to construct the sequence of the list $L_{r}$. Take the following sequence of the items for given $s \geq 1, r \geq 1$ and $k \geq 1$. A list $L_{r}$ consists of two types of rectangles. The widths of the rectangles of type $A$ are $\frac{1}{\alpha}$, and the widths of the rectangles of type $B$ are $\delta\left(0<\delta \leq \frac{1}{\alpha m}\right.$, where $m$ is a suitable multiple of $\left.t_{k}(s)-1\right)$. The sequence of the rectangles of type $A$ consists of $m \alpha(\alpha-1)(s-1)$ times rectangles with height $\frac{1}{s+1}+\varepsilon$ and another $k$ rectangles of different types with heights $\frac{1}{t_{i}(s)}+\varepsilon, 1 \leq i \leq k$, and among them there are $m \alpha(\alpha-1)$ pieces of each of the different types. Similarly, we have $m \alpha(s-1)$ pieces of the rectangles of type B bins with height $\frac{1}{s+1}+\varepsilon$ and $m \alpha$ pieces with height $\frac{1}{t_{i}(s)}+\varepsilon$ for all $1 \leq i \leq k$. It is clear that the rectangles of type A can be packed into $m(\alpha-1)$ bins, because

$$
\frac{s-1}{s}-\sum_{i=1}^{k} \frac{1}{t_{i}(s)}+O(\varepsilon)<1
$$

with a suitable small $\varepsilon>0$. The pieces from the sequence of type $B$ rectangles can be placed into one bin. Thus

$$
\operatorname{OPT}\left(L_{r}\right) \leq m(\alpha-1)+1
$$

$72 x$

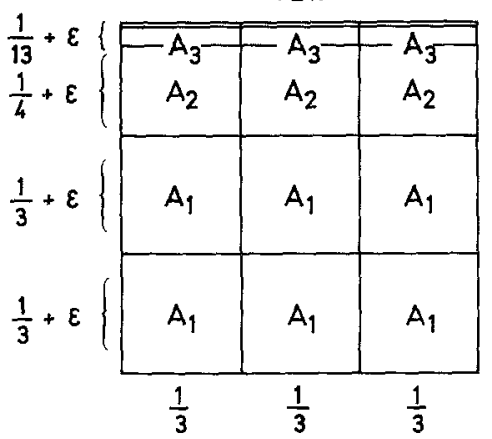

$1 x$

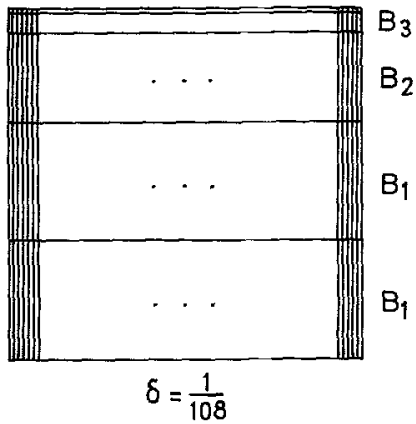

Fig. 2.3 a. An optimal packing for $s=2, r=3, k=3, \alpha=3, m=36$ 

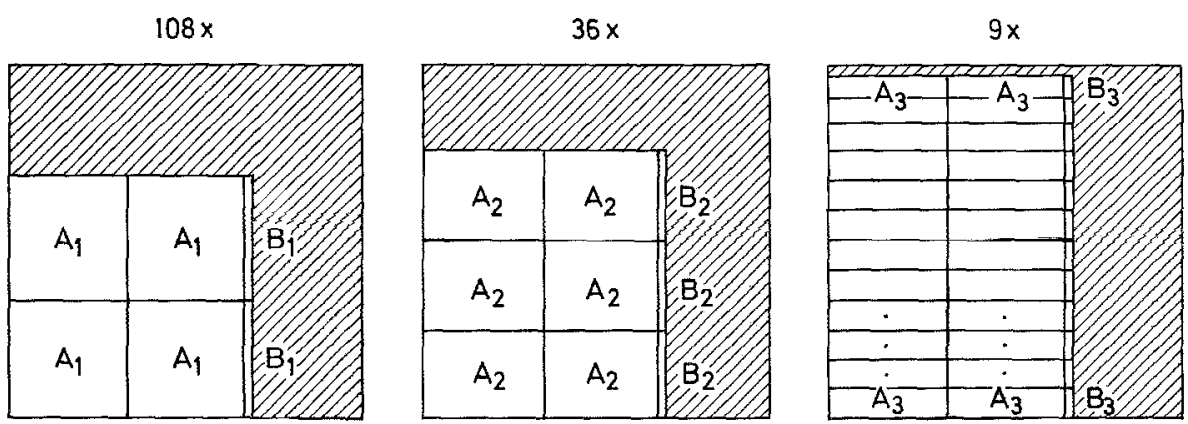

Fig. 2.3 b. The NFD packing for the worst case example $\left(\operatorname{HNF}(L) / \mathrm{OPT}(L)=\frac{153}{73}\right)$

Order the elements of the list $L_{r}$ according to the heights of the elements so that $\alpha-1$ pieces of type $A$ and one piece of type $B$ succeed each other in periodical way. Then

The ratio is

$$
\operatorname{HNF}\left(L_{r}\right) \geq m \alpha+m \alpha \sum_{i=2}^{k} \frac{1}{t_{i}(s)-1}-(k+1)
$$

$$
\frac{\operatorname{HNF}\left(L_{r}\right)}{\operatorname{OPT}\left(L_{r}\right)} \geq \frac{\alpha}{\alpha-1}\left[1+\sum_{i=2}^{k} \frac{1}{t_{i}(s)-1}-\frac{(k+1)(\alpha-1)+\alpha\left(1+\sum_{i=2}^{k} \frac{1}{t_{i}(s)-1}\right)}{m \alpha(\alpha-1)+\alpha}\right] .
$$

So the right hand side of this inequality can be made as closely to $\frac{\alpha}{\alpha-1} \gamma_{s}^{*}$ as desired by appropriate choices for $k, m$ and $\varepsilon$.

Here we give a table for the first few values of $R_{\mathrm{HNF}}\langle r, s\rangle$ :

\begin{tabular}{|c|c|c|c|c|c|}
\hline$s-r$ & 1 & 2 & 3 & 4 & 5 \\
\hline 1 & 3.382 & 3.382 & 2.536 & 2.254 & 2.114 \\
\hline 2 & 2.846 & 2.846 & 2.134 & 1.897 & 1.779 \\
\hline 3 & 2.604 & 2.604 & 1.953 & 1.736 & 1.627 \\
\hline 4 & 2.466 & 2.466 & 1.848 & 1.644 & 1.541 \\
\hline
\end{tabular}

\section{The Expected Solution Value}

In order to analyse the expected number of the bins used by the HNF heuristic we approximate its performance by that of the Sliced HNF with parameter $r\left(\mathrm{SHNF}_{r}\right)$, (see Csirik et al. [4]) in which items whose heights are larger than $\frac{1}{r}$ are packed according to the NFD rule, the last opened bin is completed to obtain at most $(r-1)$ blocks and any remaining items are packed in bins in blocks of size $r$. Contrary to the 
notations used in the previous section in this section the random variable $\mathrm{A}(n)$ will denote the number of bins used by the algorithm $A$ to pack $n$ items. Then clearly for any realization of the item sizes $(w(p), h(p)), w(p) \geq 1, h(p) \leq 1$, we obtain

and

$$
\operatorname{SHNF}_{r}(n) \geq \operatorname{HNF}(n), \quad r \geq 2, n \geq 1,
$$

$$
\lim _{r \rightarrow \infty} \operatorname{SHNF}_{r}(n)=\operatorname{HNF}(n), n \geq 1 \text {. }
$$

Consider now a sequence of positive random vectors $\left(w_{i}(p), h_{i}(p)\right)_{i=1}^{\infty}$, bounded by 1 in each component, with $\left(w_{i}(p)\right)_{i=1}^{n},\left(h_{i}(p)\right)_{i=1}^{n}$ independent subsequences consisting of independent and identically distributed random variables.

If $k_{i}(n)$ denotes the number of vectors among the first $n$ whose second component belongs to $\left(\frac{1}{i+1}, \frac{1}{i}\right]$ and $K_{i}(n)=k_{i}(n)+k_{i+1}(n)+\ldots$ then one can easily verify that

$$
\operatorname{SHNF}_{r}(n) \leq \sum_{i=1}^{r-1} \frac{\mathrm{NF}\left(k_{i}(n)\right)}{i}+\frac{\mathrm{NF}\left(K_{r}(n)\right)}{r}+r .
$$

On the other hand, if the items are packed by the HNF rule and bins containing items, whose second components are smaller than $\frac{1}{r}$ or belong to different intervals $\left(\frac{1}{i+1}, \frac{1}{i}\right], 1 \leq i \leq r-1$, are ignored, then we have that

$$
\mathrm{HNF} \geq \sum_{i=1}^{r-1} \frac{\mathrm{NF}\left(k_{i}(n)\right)}{i}-r .
$$

Hence by (3.2) and (3.3) we obtain for every fixed $r \geq 2$ immediately

$$
\sum_{i=1}^{r-1} \frac{\left.\mathrm{E}\left(\mathrm{NF}\left(k_{i}(n)\right)\right)\right)}{i} \rightarrow r \leq \mathrm{E}(\mathrm{HNF}(n)) \leq \sum_{i=1}^{r-1} \frac{\mathrm{E}\left(\mathrm{NF}\left(k_{i}(n)\right)\right)}{i}+\frac{\mathrm{E}\left(\mathrm{NF}\left(K_{r}(n)\right)\right)}{r}+r
$$

Notice that for $n, m \geq 0$

$$
0 \leq \mathrm{NF}(n+m) \leq \mathrm{NF}(n)+\mathrm{NF}(m)
$$

and so by the theory of subadditive functions (see Kingman [8])

$$
\lim _{n \rightarrow \infty} \frac{\mathrm{NF}(n)}{n}=c
$$

exist a.s. Moreover, since $\mathrm{NF}(n) \leq n$, we get by the dominated convergence theorem

$$
\lim _{n \rightarrow \infty} \frac{\mathrm{E}(\mathrm{NF}(n))}{n}=c \text {. }
$$

Using the above observations (3.4) and (3.5) and the fact that $k_{i}(n), 1 \leq i \leq r-1$, resp. $K_{r}(n)$, are binomially distributed with parameters $n, F\left(\frac{1}{i}\right)-F\left(\frac{1}{i+1}\right)$, resp. $F\left(\frac{1}{r}\right)$, where $F$ denotes the probability distribution of the height $h(p)$, we obtain by 
a standard argument

and

$$
\limsup _{n \rightarrow \infty} \frac{\operatorname{E(HNF(n))}}{n} \leq c \sum_{i=1}^{r-1} \frac{F\left(\frac{1}{i}\right)-F\left(\frac{1}{i+1}\right)}{i}+\frac{c}{r} F\left(\frac{1}{r}\right)
$$

for every $r \geq 2$.

$$
\liminf _{n \rightarrow \infty} \frac{\mathrm{E}(\operatorname{HNF}(n))}{n} \geq c \sum_{i=1}^{r-1} \frac{F\left(\frac{1}{i}\right)-F\left(\frac{1}{i+1}\right)}{i}
$$

This implies, letting $r \rightarrow \infty$

$$
\lim _{n \rightarrow \infty} \frac{\operatorname{E}(\operatorname{HNF}(n))}{n}=c \sum_{i=1}^{\infty} \frac{F\left(\frac{1}{i}\right)-F\left(\frac{1}{i+1}\right)}{i}=c \lim _{n \rightarrow \infty} \frac{\operatorname{E}(\operatorname{NFD}(n))}{n}
$$

where we get the last equation from Csirik et al. [4]. Hence we have proved the following result.

\section{Theorem 3.1:}

$$
\lim _{n \rightarrow \infty} \frac{\mathrm{E}(\mathrm{HNF}(n))}{n}=\lim _{n \rightarrow \infty} \frac{\mathrm{E}(\mathrm{NF}(n))}{n} \lim _{n \rightarrow \infty} \frac{\mathrm{E}(\mathrm{NFD}(n))}{n} .
$$

Remark: If the item sizes $\left(w_{i}(p), h_{i}(p)\right)_{i=1}^{\infty}$ are independent and uniformly distributed in the square $[0,1] \times[0,1]$ then

$$
\left.\lim _{n \rightarrow \infty} \frac{E(N F(n))}{n}=\frac{2}{3} \quad \text { (see Ong et al. }[10]\right)
$$

and

$$
\lim _{n \rightarrow \infty} \frac{\mathrm{E}(\mathrm{NFD}(n))}{n}=\left(\frac{\pi^{2}}{6}-1\right) \quad \text { (see Csirik et al. [4]). }
$$

Hence

$$
\lim _{n \rightarrow \infty} \frac{\mathrm{E}(\operatorname{HNF}(n))}{n}=\frac{2}{3}\left(\frac{\pi^{2}}{6}-1\right)
$$

and since

$$
\lim _{n \rightarrow \infty} \frac{\mathrm{E}(\mathrm{OPT}(n))}{\frac{n}{4}}=1 \text {, }
$$

this implies

$$
\lim _{n \rightarrow \infty} \frac{\mathrm{E}(\operatorname{HNF}(n))}{\operatorname{E}(\operatorname{OPT}(n))}=\frac{8}{3}\left(\frac{\pi^{2}}{6}-1\right)
$$




\section{References}

[1] Baker, B. S., Coffman, E. G. : A tight asymptotic bound for next-fit-decreasing bin packing. SIAM J. Alg. Discr. Meth. 2, 147-152 (1981).

[2] Chung, F. R. K., Garey, M. R., Johnson, D. S.: On packing two-dimensional bins. SIAM Alg. Discr. Meth. 3, 66-76 (1982).

[3] Coffman, E. G., Garey, M. R., Johnson, D. S., Tarjan, R. E.: Performance bounds for leveloriented two-dimensional packing algorithms. SIAM J. Computing 9, 808-826 (1980).

[4] Csirik, J., Frenk, J. B. G., Frieze, A. M., Galambos, G., Rinnooy Kan, A. H. G.: A probabilistic analysis of the next fit decreasing bin packing heuristic. Operations Research Letters (to appear).

[5] Garey, M. R., Johnson, D. S.: Computer and Intractability: A Guide to the Theory of NPCompleteness. San Francisco: W. H. Freeman 1979.

[6] Johnson, D. S.: Fast algorithms for bin-packing. System Sci. 8, 272-314 (1974).

[7] Johnson, D. S., Demers, A., Ullman, J. D., Garey, M. R., Graham, R. L.: Worst-case performance bounds for simple one-dimensional packing algorithms. SIAM J. Computing 3, 256-278 (1974).

[8] Kingman, J. F. C.: Subadditive Processes, Lecture Notes in Mathematics. Springer-Verlag, 539, $168-222(1976)$.

[9] Liang, F. M.: Lower bound for on-line bin packing. Information Proc. Lett. 10, 76-79 (1980).

[10] Ong, H. L., Magazine, M. J., Wee, T. S.: Probabilistic analysis of bin packing heuristics. Operations Research 32, 983-998 (1984).

J. B. G. Frenk

Faculteit der Economische Wetenschappen

Erasmus Universiteit

Postbus 1738

NL-3000 DR Rotterdam

The Netherlands
G. Galambos

Kalmár Laboratory of Cybernetics

József Attila University

Árpád tér 2

H-6720 Szeged

Hungary 